\title{
Role of MRI in the evaluation of acoustic schwannoma and its comparison to histopathological findings
}

\author{
Haque $\mathrm{S}^{1}$, Hossain $\mathrm{A}^{2}$, Quddus $\mathrm{MA}^{2}$, Jahan $\mathrm{MU}^{3}$ \\ ${ }^{I}$ National Institute of Cancer Research \& Hospital, Dhaka, ${ }^{2}$ Department of Radiology, Dhaka Medical \\ College, Dhaka, ${ }^{3}$ Department of Radiology \& Imaging, Bangabandhu Sheikh Mujib Medical University, Dhaka.
}

\begin{abstract}
Magnetic Resonance Imaging (MRI) has been the primary imaging modality and has revolutionized the imaging of brain tumors. MRI can display accurate multi planer imaging without interfering of adjacent structures specially for posterior fossa mass lesion. MRI is the imaging modality of choice for cerebollo-pontine (CP) angle Schwannoma. The study was performed to determine, the diagnostic accuracy of MRI in the evaluation of intracranial extra axial CP angle Schwannoma. MRI scan of brain was done on 42 consecutively selected patients referred for the evaluation of CP Acoustic Schwannoma. The age range from 21-60 years and the mean age was $42.85( \pm 9.5)$ years. Highest incidence of cerebollo-pontine angle (CPA) mass were found 42.86\% in 41-50 age group of patients. Male and Female ratio was 1.083:1. The most common presenting feature of the patients with CP angle Acoustic Schwannoma were headache $90.48 \%$. Acoustic Schwannoma is $\mathrm{T}_{1}$ hypointense $100 \%, \mathrm{~T}_{2}$ hyper intense $84.61 \%$ and heterogeneously hyper intense $92.30 \%$ in FLAIR image. After giving contrast agents, homogeneous enhancement $57.69 \%$ and heterogeneous $42.31 \%$ cases of Acoustic Schwannoma. Overall 61.54\% Acoustic Schwannoma strong contrast enhancement was observed. Dural tail was observed in $26.92 \%$ cases. Perilesional edema was observed $38.46 \%$ cases. Mass effect was observed in $76.92 \%$. After complete MRI evaluation 61.9\% had Acoustic Schwannoma. Histopathologicaly proved cases showed out of all patients Acoustic Schwannoma 59.52\%. The overall sensitivity of MRI to diagnose Acoustic Schwannoma were found, Sensitivity- 96\%, Specificity88.2\%, PPV-92.31\%, NPV-93.75\% and Acceuracy 92.86\%. Test is significant with $\mathrm{p}<0.0001$ level. It is conceivable that MRI is a highly accurate, sensitive and Gadolinium enhanced MRI is more sensitive in detection of acoustic Schwannoma. MR imaging is the study of choice for the examination of the patient of cerebellopontine angle Schwannoma because of its high sensitivity specially after use of contrast material.
\end{abstract}

\section{Introduction}

Intra-cranial tumors are the most devastating illness in human being. In the developed world, cerebral tumors account for $2 \%$ all death at all ages ${ }^{1}$. The incidence of Primary intracranial cerebollo-pontine (CP) angle Schwannoma account approximately $8-10 \%$ of all neoplasms. The most common cerebollo-pontine angle (CPA) mass is Acoustic Schwannoma. Among the CPA masses, vestibular Schwannoma accounts $75 \%$ of the lesions ${ }^{2}$. With the advent of Computed Tomography (CT) and Magnetic Resonance Imaging (MRI) there is revolutionary change in the detection of intracranial tumor $^{3}$. Now the availability and cost effectiveness with greater accuracy and fewer false negative cases, MRI has become the prime modality of investigation to detect intracranial tumors specially Acoustic Schwannoma. In MRI, multi planner imaging, cross sectional anatomical detail sagittal coronal axial reformat with contrast and FLAIR images plays excellent role and remains as a major imaging technique for detection and localization of CPA Acoustic Schwannoma ${ }^{2}$. Modern 1.5 Tesla or above high resolution MRI with Diffusion Weighted image and Perfusion Weighted image and gradient Echo, FLAIR images will localize and characterize the vast majority of cerebello-pontine angle Acoustic Schwannoma .

This study assess the effectiveness of MRI for the evaluation of CPA Acoustic Schwannoma and its comparison with histopathological findings. It also elucidate the accuracy, sensitivity, specificity of MRI in detection of acoustic Schwannoma.

\section{Materials and Methods}

This cross sectional prospective observational study was carried out on consecutively selected 47 patients ranging from 21-60 years of age referred for MRI scan of brain with a clinical suspicion of 
CP angle mass in the Department of Radiology and Imaging Dhaka Medical College Hospital (DMCH), Dhaka in collaboration with the department of Neurosurgery and Pathology of same Hospital from July 2008 to March 2010. At first all the patients were evaluated by detail history and clinical examination with special emphasis on nervous system. Subsequently MRI scan of brain was performed in all cases. Patients who were operated continuously followed after the surgery up to histo-pathological diagnosis were made. The histopathological reports were collected thus, were compared with MRI findings. All these information's were collected in pre-designed structured data collection sheets. Inclusion criteria was suspected cases of cerebello-pontine angle masses referred to the department of Radiology and Imaging DMCH from Out Patient Department (OPD) or Indoor. Patients who were unfit for surgery or nor willing to undergo surgery also Patients in whom histopathological report were unavailable, were excluded from the study.

\section{Result}

The main objective of the study was to establish the diagnostic usefulness of MRI in detection of $\mathrm{CP}$ Angle Schwannoma.

A total number of 42 patients who were clinically suspected, having $\mathrm{CP}$ angle cistern mass were included consecutively in this study. The age range was from 21-60 years. The highest incidence of CPA masses was found in $42.86 \%$ was found in the age between 41-50 years age group and followed by $51-60$ years of age group $23.81 \%$ and $21.43 \%$ in $31-40$ years age group and $11.90 \%$ at the age $21-30$ years age group.

Table I: Age distribution of the respondents $(n=42)$

\begin{tabular}{lll}
\hline Age group (years) & Frequency & Percent \\
\hline $21-30$ & 5 & 11.90 \\
$31-40$ & 9 & 21.43 \\
$41-50$ & 18 & 42.86 \\
$51-60$ & 10 & 23.81 \\
\hline Total & 42 & 100.00 \\
\hline Mean \pm SD & \multicolumn{3}{c}{$42.85 \pm 9.50$} \\
\hline
\end{tabular}

Out of 42 respondents (22) $52 \%$ was male and (20) $48 \%$ was female. Male and female ratio is 1.0833 .

Table II: Sex distribution of the respondents $(n=42)$

\begin{tabular}{lll}
\hline Sex & Frequency & Percent \\
\hline Male & 22 & $52 \%$ \\
Female & 20 & $48 \%$ \\
\hline Total & 42 & $100 \%$ \\
\hline Mean \pm SD & & $21.0 \pm 0.21$ \\
\hline
\end{tabular}

The most common presenting feature associated with CPA tumor was headache which was observed in 38 patients $(90.48 \%)$. Another common symptom was tinnitus $80.95 \%$, Vertigo $61.90 \%$ and $52.38 \%$ complained of hearing loss than followed by disequilibrium $40.48 \%$ and facial weakness $21.43 \%$.

Table III: Distribution of the respondents by clinical feature $(n=42)$

\begin{tabular}{lll}
\hline Clinical feature & Frequency & Percent \\
\hline Tinnitus & 34 & 80.95 \\
Hearing loss & 22 & 52.38 \\
Disequilibrium & 17 & 40.48 \\
Vertigo & 26 & 61.90 \\
Facial weakness & 9 & 21.43 \\
Headache & 38 & 90.48 \\
Exopthalmus & 5 & 11.90 \\
Wide gait & 7 & 16.67 \\
\hline
\end{tabular}

Table IV: MRI features of Acoustic Schwannoma ( $\mathrm{n}=26)$

\begin{tabular}{llcl}
\hline MRI findings & Characteristics & No of cases & Percent \\
\hline Signal intensity & $\mathrm{T}_{1}$ WI- Hypointense & 26 & $100 \%$ \\
& $\mathrm{~T}_{2}$ WI- Hyper intense & 22 & $84.61 \%$ \\
& FLAIR: heterogenously & & \\
& hyper intense & 24 & $92.30 \%$ \\
Contrast & Homogeneous & 15 & $57.69 \%$ \\
Enhancement & Heterogeneous & 11 & $42.30 \%$ \\
Enhancement & Strong & 16 & $61.53 \%$ \\
Character & Moderate & 10 & $38.46 \%$ \\
Mass effect & & 20 & $76.92 \%$ \\
Perilesional & & 10 & $38.46 \%$ \\
oedema & & 7 & $26.92 \%$ \\
Dural tail & & & \\
Involvement of & & 19 & $73.07 \%$ \\
internal acoustic & & & \\
canal & & &
\end{tabular}

Table-IV shows the different MRI features of Acoustic schwannoma. Out of all tumors $100 \%$ were hypointense in $\mathrm{T} 1 \mathrm{~W} 1$ and $84.61 \%$ hyper intense in $\mathrm{T} 2 \mathrm{~W} 1$ and $92.30 \%$ tumors were heterogeneously hyper intense in FLAIR image

After giving contrast agents, homogeneous enhancement was observed in $57.69 \%$ cases and heterogeneous in $42.30 \%$. Enhancement was strong in $61.53 \%$ and moderate enhancement in $38.46 \%$ cases. $76.92 \%$ had mass effect, perilesional edema $38.46 \%$ cases. Dural tail was observed in $26.92 \%$ and involvement of Internal Acoustic Canal (IAC) in $73.07 \%$ cases. Out of 42 patients $21(50 \%)$ tumor was mixed in nature, $18(42.86 \%)$ solid and 3 (7.14\%) was cystic in nature. Out of all cases 24 was diagnosed as AS by MRI and Confirmed by histopatholigical evaluation they were true positive. 2 case was diagnosed as Acoustic Schwannoma (AS) by MRI, but not confirmed by histopathological findings. They were false positive. Of 16 cases of other than Acoustic Schwannoma which were confirmed by MRI, 1 
was confirmed as Acoustic Schwannoma and 15 were other than AS by histopathology. They were false negative and true negative respectively.

Table V: MRI findings and Histopathological findings of schwannoma: $(n=42)$

\begin{tabular}{cccc}
\hline \multirow{2}{*}{$\begin{array}{c}\text { Test Result } \\
\text { MRI }\end{array}$} & \multicolumn{2}{c}{ True state of patients } & Total \\
Diagnosis & \multicolumn{2}{c}{ Histological diagnosis } & \\
\cline { 2 - 3 } & True & False & \\
& schwannoma & schwannoma & \\
\hline Positive & True Positive & False Positive & Patients with \\
& (TP) & positive \\
& 24 & 2 & test (TP + FP) \\
Negative & False Negative & True Negative & Patients with \\
& (FN) & (TN) & Negative \\
& 1 & 15 & test (FN + TN) \\
Total & All patients & False & All Patient \\
& with true & schwannoma & studied \\
& schwannoma & (FP+TN) & (TP+FP+FN+T \\
& (TP+FN) & 17 & N) \\
& 25 & & 42 \\
\hline
\end{tabular}

Sensitivity of MRI diagnosis for schwannoma was $96 \%$, specificity $88.24 \%$, positive predicative value $92.31 \%$, negative predicative value $93.75 \%$ and accuracy $92.86 \%$. Chi-square test results $\chi^{2}$ $=31.695$ with 3 degree of freedom. The two tailed $p$ value is less than 0.0001 by conventional criteria this $p$ value is considered to be extremely statistically significant.

\section{Discussion}

The diagnostic approach to neurological problems has undergone significant change with the introduction of Magnetic Resonance Imaging MRI has proven to be an excellent technique for visualization of the posterior fossa and CP Angle tumor.Magnetic Resonance Images can be acquired with equal clarity in any orientation, axial, sagittal, coronal, image, artifacts from bone are absent with MRI. MRI is the imaging modality of the choice for CPA and internal auditory canal masses ${ }^{5}$. MRI should be used complementarily in CPA diagnostics ${ }^{5}$.

Gadolinium di-ethylene tri amino penta acetic acid (Gd-DTPA) enhanced MRI imaging improves the reliability of CPA mass diagnosis. Schwannomas are more contrast enhancing than Meningiomas. Big Schwannomas presented mostly heterogeneous enhancement caused by the cystic degeneration, necrosis and hemorrhage. ${ }^{5}$. MRI imaging is the study of choice for the examination of patients with suspected Schwannoma, because of its high sensitivity, especially after the use of contrast material $^{3}$. A large variety of unusual lesion can be encountered in the CPA and should be differentiated from acoustic neuroma and meningioma. Signal intensity at MRI imaging enhancement, shape and margins, extent, mass effect is helpful in establishing the diagnosis ${ }^{6,12}$. This study was carried out with an aim to establish the usefulness of MRI in detection of acoustic Schwannoma and to compare the post operative histopathological diagnosis of CPA Schwannoma with the MRI along with its validity tests by calculating sensitivity, specificity, accuracy, positive predictive value (PPV) and negative predictive value (NPV) respectively of MRI.

In this study the mean age of the patient was $42.85 \pm 9.25$ years of age range from 21-60 years. Highest incidence of CPA mass was $42.86 \%$ found in $41-50$ years age group followed by $23.81 \%$ in 51-60 years age group.

Regarding the Acoustic schwannoma Mulkens et al. (1993) worked with 81 patients of Acoustic Schwannoma in which the mean age was 54 years ${ }^{3}$. The age range of the present study which is almost similar that of the study of Mulkens et $\mathrm{al}^{3}$. Similarly Osborn (1994) has mentioned the peak occurrence of Acoustic Schwannoma between 4060 years $^{13}$.

In our series out of all respondents $52 \%$ were male and $48 \%$ were female. According to Osborn ${ }^{13}$ (1994) it has a slightly female preponderance (1.5:1-2:1) which contradicts to our study, where we found that ratio of male and female was almost equal $^{2,9}$.

Symptoms and signs of brain tumor can result from the focal effects of the tumor on neighboring areas. The symptoms of CPA masses arise from compression of local structures within as at the boundaries of the region, compression of fibres of cochlear nerve may result in sensory hearing loss ${ }^{1}$. The most common presenting symptoms headache $90 \%$, tinnitus $80 \%$. 26 cases of $\mathrm{AN}$ - hearing loss may be sudden, and tinnitus was found in $56 \%$ cases. Clinical presentation of CPA masses were found tinnitus, hearing loss, disequilibrium and visual disturbances. In the present study the most common presenting symptom was headache, sensory hearing loss, tinnitus and visual disturbances which is almost same with the previous study ${ }^{1,5,12}$.

In the present series Acoustic-Schwannoma was hypointense in $\mathrm{T}_{1} \mathrm{WI}$ in all cases (100\%) and hyper intense in $\mathrm{T}_{2} \mathrm{WI}$ 22(84\%). In FLAIR heterogeneously hyper intense $24(92 \%)$ cases contrast enhancement was homogeneous in 15 $(57 \%)$ cases and heterogeneous in $11(43 \%)$ cases.

Pattern of enhancement was strong in 16(61\%) cases and moderate was 10 (39\%) cases. Mulkens et $\mathrm{al}^{3,4}$ (1993) has found 35\% of tumors was iso intense to brain, $63 \%$ were hypointense in T1W1 
and after contrast all tumors showed intense enhancement $^{3,7}$. It was homogeneous in $67 \%$ and inhomogeneous $10 \%$ and heterogeneous with areas of cystic degeneration in $22 \%$. Osborn (1994) has mentioned that $70 \%-75 \%$ tumors specially Acoustic Schwannoma were hypointense to brain and $25 \%$ was iso intense, tumors were hyper intense in $\mathrm{T}^{2} \mathrm{WI}^{13}$. Almost homogeneous enhancement $67 \%$ and mildly inhomogeneous $10 \%$ cases and heterogeneous in $22 \%$ which show similar result with the present study ${ }^{2}$.

In the present series it was observed that mass effects were present in 20(76\%) cases of Schwannoma. MR imaging has rapidly supplemented other imaging techniques in the diagnosis of these tumors, especially after the development of paramagnetic contrast agents. The use of contrast material improves the sensitivity of MR imaging by selectively increasing the level of contrast enhancement in all acoustic schwannomas ${ }^{7,8}$. The capillaries of these extra-axial tumors do not exhibit a blood brain barrier and theirs degree of enhancement is greater that of any other intra-cranial tumor ${ }^{3}$. 39\% of Acoustic schwannoma $T_{2}$ weighted images mild to moderate peritumoral edema was visible.

Mulkens et al. (1993) showed 13(37\%) of the $35 \mathrm{~T}_{2}$ WI- mild of moderate peritumoral edema was visible in AN which is compatible with present study ${ }^{3}$.

Dural tail was found in 7 out of $26(26 \%)$ schwannoma. It is an important sign in the differential diagnosis of tumor centered at the meatus of the internal auditory canal and or with an "acute angle" with the petrous bone is more likely to be an acoustic schwannoma. In our study we found that 19 out of $26(73 \%)$ Acoustic Schwannoma has an involvement to internal acoustic canal. The angle formed between the tumor border and petrous bone was acute in $81 \%$ which is quite similar with present study. From the result of present study as well as the findings obtained by other study ${ }^{4}$, it is conceivable that MRI is a highly accurate and sensitive and Gadolinium enhanced MRI is more sensitive in detection of Acoustic Schwannoma ${ }^{8,9}$. MRI imaging is the study of choice for the examination of patients with suspected CPA masses because of its high sensitivity specially after use of contrast material.

Sensitivity of MRI diagnosis for schwannomas was $96.00 \%$, specificity $88.24 \%$, positive predicative value $92.31 \%$, negative predicative value $93.75 \%$ and accuracy $92.86 \%$. Mulkens et al.(1993) have found sensitivity for Schwannoma $98.00 \%$ accuracy $83.33 \%$, PPV $92.77 \%^{3}$. This results are strongly comparable with that of results of present study.

Swieszewska: et al. (2006) found overall sensitivity specificity and accuracy of cerebello pontine angle masses were $86.52 \%, 80.00 \%$ and $84.87 \%$ respectively ${ }^{5}$. In the present study the results were found to be comparable with this results mentioned above 5 .

From the results of present study as well as the findings obtained by Smirniotopoulos, Yue and Rusbing (1993), it is conceivable that MRI is a highly accurate and sensitive modality in the evaluation of cerebello pontine angle Schwannoma ${ }^{4}$. So MR Imaging is the study of Choice for examination of patients with suspected CP Angle Schwannoma.

Conclusion: MRI findings of the present study correlated well the histo pathological results. It can therefore be concluded that MRI is useful modality and study of choice in the diagnosis of CPA Schwannoma because of its high sensitivity and specificity specially after the use of contrast. MRI thus can be regarded as an important imaging modality in the diagnosis of cerebello pontine Angle Acoustic Schwannoma ${ }^{1,3,5}$.

\section{References}

1. Alane, L Cowan, Gadre, Arun, B\& Ryan, Mathew Cerebellopontine Angle Masses, Grand Rounds Presentation UT MB Dept. of Otolaryngology, University Texas, Medical Branch, 2004; June 2.

2. Rauschning, Woltgang. Brain tumors and tumor like masses, classification and differential diagnosis in Diagnostic Neuro Radiology, eds Osborn, AG, Elsevier Publishing, India, 1994; 438-439, 441, 593, 626-633.

3. Mulkens, T H, Parizel P m, Martin J J, Degryse, H.R, Heyning, P.H \& Forton, G E. Acoustic Schwannoma MR Findings in 84 tumor. American Journal of Radiology 1993; 160: 395-398.

4. Smirniotopoulos, James G, Nancy, Chang \& Rusbing, Elisabeth. Cerebellopontine Angle Masses Radiologic Pathologic Correlation. Radiographics 1993; 13: 11311147.

5. Swieszewska, Ewa Izycka, Szurowska, Edyta, Kloc, Wojciech, Rzepko, Rubert, Wybieralska, Mirostawa, Dubaniewicz \& Skurek, Andzej. Cerebello pontine angle tumors radiologic-pathologic correlation. Folia Neuropathol 2006; 44(4): 274-281.

6. Bonneville, Fabrice, Sarrazin, Tean-JLuc, Dupuch, MK, Iffenecker, C, Cordoliani \& YS Doyun, Dominique. Unusual Lesions of the Cerebellopontine Angle: A segmental Approach. Radiographics 2001; 21: 419-438. 
7. Rauschning Woltgang. Brain tumors and tumor like masses, classification and differential diagnosis in Diagnostic Neuroradiology, eds Osborn, AG, Elsevier Pulishing, India, 1994; 438-439, 441, 593, 626-633.

8. Sarrazin I, O \& Helie, Cordoliani Y. Cerebellopontine Angle Tumors in adults. Journal de radiologie 2000; 81: 675-90.

9. Kieffer, Stephen A \& Brace, Jeffrey R. Intracranial neoplasms in CT MRI of Whole body eds Haaga, JR, Dogra, SV, Forsting, MGilkeson, RC \& Sundaram, M, Mosby ELSEVIER, Philadelphia, 2003; 95-99.

11. Stuckey SL, Harries AJ \& Mannolini M. Detection of Acoustic Schwannoma: Use of Constructive Interference in the steady state three dimensional MR. American Society of Neuro Radiology 1996; 17: 12191225.
12. Yoshionori, Shigamatsu, Yukunori, Korogi, Toshinori, Hirai, Tomoko, Okuda, Ichiro, Ikushima, Takeshi, Sugahara \& Luxia, Liang. Contrast enhanced CISS MRI of vestibular Schwannomas: Phantom and clinical studies. Neuro Radiology 1999; 23: 224-231.

13. Osborn AG. Miscellanies Tumors, Cyst and Metastses in Diagnostic Neuroradiolog, Elsevier Publishing, India, 1994; 626-633.

10. Brors, D Schafers M, Bodmer D, Draf, W Kahle \& G, Schick B. Post operative magnetic resonance imaging findings after trans temporal and trans labyrinthine vestibular schwannoma resection. The laryngoscope 2003; 113(3): 420-428. 